\title{
Floquet formulation for the investigation of multiphoton quantum interference in a superconducting qubit driven by a strong ac field
}

\author{
Sang-Kil Son (손상길), ${ }^{1, *}$ Siyuan Han (韩思远), ${ }^{2, \dagger}$ and Shih-I Chu (朱時宜) $)^{1, \#}$ \\ ${ }^{1}$ Department of Chemistry, University of Kansas, Lawrence, Kansas 66045, USA \\ ${ }^{2}$ Department of Physics, University of Kansas, Lawrence, Kansas 66045, USA
}

(Received 3 August 2008; published 3 March 2009)

\begin{abstract}
We present a Floquet treatment of multiphoton quantum interference in a strongly driven superconducting flux qubit. The periodically time-dependent Schrödinger equation can be reduced to an equivalent timeindependent infinite-dimensional Floquet matrix eigenvalue problem. For resonant or nearly resonant multiphoton transitions, we extend the generalized Van Vleck (GVV) nearly degenerate high-order perturbation theory for the treatment of the Floquet Hamiltonian, allowing the reduction of the infinite-dimensional Floquet matrix to an $N \times N$ effective Hamiltonian, where $N$ is the number of eigenstates under consideration. The GVV approach allows accurate treatment of ac Stark shift, power broadening, time-dependent and time-averaged transition probability, etc., well beyond the rotating wave approximation. We extend the Floquet and GVV approaches for numerical and analytical studies of the multiphoton resonance processes and quantum interference phenomena for the superconducting flux qubit system $(N=2)$ driven by intense ac fields.
\end{abstract}

DOI: 10.1103/PhysRevA.79.032301

PACS number(s): 03.67.Lx, 85.25.-j, 42.50.Hz

\section{INTRODUCTION}

Superconducting flux qubit [1] is a promising candidate for quantum computing [2]. When the superconducting qubit is driven by an oscillating field, it involves plentiful dynamics of macroscopic quantum states such as Rabi oscillations and coherent control $[3,4]$. Recently, Oliver and colleagues experimentally demonstrated multiphoton resonance and quantum interference in a strongly driven flux qubit as an analogue of Mach-Zehnder interferometry $[5,6]$. The peaks in the transition probability between two qubit states form fringe patterns around multiphoton resonance positions. This is explained by the phase differences of the qubit states after they are swept by a strong driving field [5]. Since the strongly driven qubit can be modeled by a two-level system interacting with the strong oscillating field, the development of an analytical approach for a time-dependent problem is desirable to explore the rich dynamics of the two-level system. Understanding dynamics of strongly driven two-level systems is important to superconducting approach of quantum computation. For instance, because superconducting qubits usually have short coherence time [3,4,7-9], it is often necessary to apply the strong ac field to decrease the time required for each gate operation. Furthermore, microwave spectroscopy, especially the position of resonant peaks, is also widely used to calibrate qubit parameters which are crucial to reduce gate errors [3,4,7-9]. Therefore, treating strongly driven qubits is not only theoretically desirable but also practically useful.

The generalized Floquet formalisms [10] have been developed and applied to a number of time-dependent problems: from two-level models to realistic atomic and molecular systems [10-13], and from monochromatic to

\footnotetext{
*sangkil@ku.edu

†han@ku.edu

sichu@ku.edu
}

polychromatic [14] and frequency-comb laser fields [15]. It provides nonperturbative and accurate treatment of the interaction of a quantum system with intense time-dependent fields. In the Floquet procedure, a time-dependent problem can be transformed into an equivalent time-independent infinite-dimensional Floquet matrix eigenvalue problem. For analytic treatment of an $N$-level system, this infinitedimensional matrix can be further reduced into an effective $N \times N$ matrix. The rotating wave approximation (RWA) includes only one resonant term, whereas the generalized Van Vleck (GVV) nearly degenerate perturbation theory $[16,17]$ includes all leading terms that result in the ac Stark level shift and power broadening of multiphoton resonance positions.

The two-level system that interacts with the external field through off-diagonal time-dependent couplings (i.e., transverse couplings) has been extensively studied in the framework of the Floquet theory $[10,17,18]$. On the contrary, the qubit that is the two-level system interacting through diagonal couplings (i.e., longitudinal couplings) with driving fields has been investigated only within RWA [5,19]. Recently, Ashhab et al. [20] have studied the two-level system within RWA and the transfer-matrix approach, and Greenberg [21] and Wilson et al. [22] have studied the qubit using the dressed-state approach. However, to the best of our knowledge, the ac Stark level shift for multiphoton resonance processes which appears beyond RWA has not been considered so far for this qubit system. As to be shown later, the consideration of level shift and power broadening is significant for quantitative exploration of multiphoton quantum interference phenomena in the superconducting qubit. In this paper, we extend the Floquet theory for a comprehensive investigation of the strongly driven qubit system and provide insightful GVV analytic solutions to better understand the multiphoton resonance condition including the level shift and coherent quantum interference patterns.

The outline of the paper is as follows. In Sec. II, we present the generalized Floquet formalism for a supercon- 


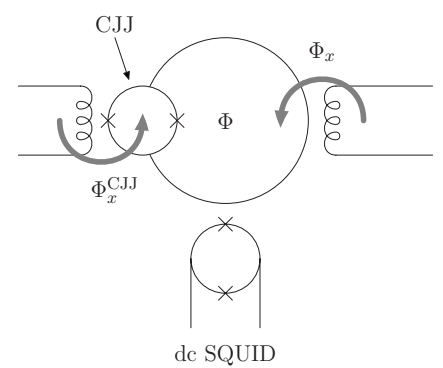

FIG. 1. Schematic diagram for a combined Josephson junction rf SQUID.

ducting flux qubit system driven by a strong ac field. In Sec. III, we present analytic GVV solutions for level shift and transition probability, etc., for the analysis of multiphoton quantum interference. In Sec. IV, we compare the numerical results of the full Floquet matrix and analytic results of RWA and GVV, and show quantum interference fringe patterns in the transition probability. This is followed by conclusion in Sec. V.

\section{FLOQUET FORMULATION FOR A SUPERCONDUCTING FLUX QUBIT}

The superconducting flux qubit discussed here is a compound Josephson junction (CJJ) rf superconducting quantum interference device (SQUID) as shown in Fig. 1 [23]. It consists of a main loop and a CJJ loop subjected to external flux biases $\Phi_{x}$ and $\Phi_{x}^{C J J}$, respectively. The CJJ loop is interrupted by two nominally identical Josephson tunnel junctions connected in parallel with total capacitance $C$ and critical current $I_{c}$. The CJJ and main loops have inductances $L_{\mathrm{CJJ}}$ and $L$, respectively. For $L_{\mathrm{CJJ}} \ll L$, the Hamiltonian of the CJJ rf SQUID can be approximated by that of a simple rf SQUID as

$$
H=\frac{Q^{2}}{2 C}+U(\Phi)
$$

where $\Phi$ represents the total flux threading the main loop and $Q$ is the charge stored in the capacitance. The potential energy of the rf SQUID is

$$
U(\Phi)=\frac{\left(\Phi-\Phi_{x}\right)^{2}}{2 L}-E_{J} \cos \left(\frac{\pi \Phi_{x}^{C J J}}{\Phi_{0}}\right) \cos \left(\frac{2 \pi \Phi}{\Phi_{0}}\right),
$$

where $E_{J}=\Phi_{0} I_{c} / 2 \pi$ and $\Phi_{0} \equiv h / 2 e$ is the flux quantum. For $\beta_{L}\left(\Phi_{x}^{\mathrm{CJJ}}\right) \equiv\left(2 \pi L I_{c} / \Phi_{0}\right) \cos \left(\pi \Phi_{x}^{\mathrm{CJJ}} / \Phi_{0}\right) \gtrsim 1, \quad U(\Phi)$ is a double-well potential and thus this device can be operated as a qubit for $-\Phi_{0} / 2 \leqslant \Phi_{x}^{\mathrm{CJ}} \leqslant \Phi_{0} / 2$ and $\Phi_{x} \approx \Phi_{0} / 2$. Denoting the counterclockwise as the positive direction of the persistent current $I_{p}$ in the loop, the two logic states of the qubit, $|\alpha\rangle$ and $|\beta\rangle$, correspond to $I_{p}<0$ and $I_{p}>0$ and thus the flux particle in the left- and right-hand wells, respectively. The state-dependent persistent current is given by $I_{p}=\langle\alpha|(\Phi$ $\left.-\Phi_{x}\right) / L|\alpha\rangle$ or $\left\langle\beta\left|\left(\Phi-\Phi_{x}\right) / L\right| \beta\right\rangle$. If the temperature is much less than the small oscillation frequency (i.e., the plasma frequency) at the bottom of the double-well potential the superconducting flux qubit can be described by a $2 \times 2$ effective Hamiltonian matrix [24],

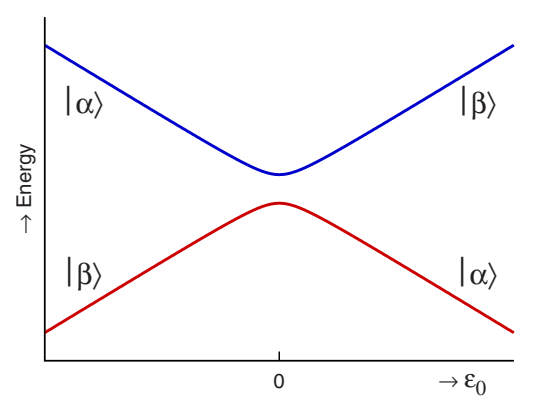

FIG. 2. (Color online) Energy diagram for a superconducting flux qubit.

$$
H_{0}=-\frac{1}{2}\left(\begin{array}{cc}
\varepsilon_{0} & \Delta \\
\Delta & -\varepsilon_{0}
\end{array}\right)
$$

where $\Delta$ is called the tunnel splitting and $\varepsilon_{0}$ is the detuning energy given by $\varepsilon_{0}=2\left|I_{p}\right| \delta \Phi_{x}$ where $\delta \Phi_{x} \equiv \Phi_{x}-\Phi_{0} / 2$ is the flux detuning. Note that Eq. (3) is obtained using the two logic states of the qubit, $|\alpha\rangle=\left(\begin{array}{ll}1 & 0\end{array}\right)^{T}$ and $|\beta\rangle=\left(\begin{array}{ll}0 & 1\end{array}\right)^{T}$, as the bases. Figure 2 shows the eigenvalues of Eq. (3), $E$ $= \pm \sqrt{\varepsilon_{0}^{2}+\Delta^{2}} / 2$, as a function of the detuning energy $\varepsilon_{0}$. A positive slope of the dispersion curve corresponds to $|\beta\rangle$ while a negative slope to $|\alpha\rangle$. These two states are coupled through the tunneling strength $\Delta$ and show the avoided crossing at $\varepsilon_{0}=0$ as shown in Fig. 2. Thus, $\Delta$ is given by the energy separation of two eigenstates of $H_{0}$ at $\varepsilon_{0}=0$ which are $(|\alpha\rangle-|\beta\rangle) / \sqrt{2}$ and $(|\alpha\rangle+|\beta\rangle) / \sqrt{2}$. Both $\left|I_{p}\right|$ and $\Delta$ are controlled in situ by $\Phi_{x}^{\mathrm{CJJ}}$. Note that $\left|I_{p}\right|$ is a decreasing function of $\Phi_{r J}^{\mathrm{CJ}}$ while $\Delta$ increases from zero to the plasma frequency as $\Phi_{x}^{\text {CJJ }}$ approaches $\pm \Phi_{0} / 2$. For $\Phi_{x}^{\text {CJJ }}=0$ one expects $\Delta \rightarrow 0$. When the qubit becomes localized in $|\alpha\rangle$ or $|\beta\rangle I_{p}$ generates a magnetic flux that can be resolved by an inductively coupled dc SQUID (see Fig. 1) as described in Ref. [23].

When the superconducting flux qubit is driven by a strong ac field, the time-dependent Hamiltonian is given as [5]

$$
H(t)=-\frac{1}{2}\left(\begin{array}{cc}
\varepsilon(t) & \Delta \\
\Delta & -\varepsilon(t)
\end{array}\right)
$$

where $\varepsilon(t)=\varepsilon_{0}+A \cos \omega t$. Note that for typical superconducting flux qubits the longitudinal coupling is more commonly used than the transverse coupling. $\omega$ is the angular frequency of the driving ac field and $A$ is its amplitude that is parametrized in the energy unit and proportional to ac flux bias [6]. Note that this Hamiltonian generally describes the two-level system interacting through the longitudinal coupling and is directly applicable to other qubit systems. For example, in Cooper-pair box qubits [22] the electrostatic energy and the Josephson coupling energy directly correspond to $\varepsilon_{0}$ and $\Delta$ in Eq. (4), respectively.

The generalized Floquet theory [10] provides an exact formulation of time-periodic problems and a combined picture of the $N$-level system and electromagnetic fields by the use of quasienergy states. According to the Floquet theorem [25], for a given time-periodic Hamiltonian, the timedependent Schrödinger equation, 


$$
i \hbar \frac{\partial}{\partial t} \psi(t)=H(t) \psi(t)
$$

has a solution that can be written as

$$
\psi(t)=e^{-i q t / \hbar} \phi(t),
$$

where $\phi(t)$ is periodic in time and $q$ is called the quasienergy. When Eq. (6) is substituted into Eq. (5), we obtain an eigenvalue equation for the quasienergy,

$$
\left(H(t)-i \hbar \frac{\partial}{\partial t}\right) \phi(t)=q \phi(t) .
$$

The periodically time-dependent problem can be transformed into an equivalent time-independent infinitedimensional generalized Floquet matrix eigenvalue problem $[10,18]$. The temporal part of the Hamiltonian $H(t)$ and the quasienergy eigenfunction $\phi(t)$ is expanded with the Fourier components of $\omega$,

$$
\begin{aligned}
& H(t)=\sum_{n} H^{[n]} e^{-i n \omega t}, \\
& \phi(t)=\sum_{n} \phi^{[n]} e^{-i n \omega t},
\end{aligned}
$$

where $H^{[n]}$ and $\phi^{[n]}$ are spanned by any orthonormal basis set. We can employ the Floquet-state nomenclature [10],

$$
|\alpha n\rangle=|\alpha\rangle \otimes|n\rangle,
$$

where $\alpha$ is the system index and $n$ is the Fourier index that runs from $-\infty$ to $\infty$. Note that in the generalized Floquet formalism $\alpha$ can be the $N$-level system index, but for the effective Hamiltonian of Eq. (4) it is restricted to the qubit state $(N=2)$. Substituting Eqs. (8) and (9) into Eq. (7) and employing Eq. (10), we obtain the following timeindependent Floquet matrix eigenvalue equation:

$$
\sum_{\beta} \sum_{m}\left\langle\alpha n\left|H_{F}\right| \beta m\right\rangle\left\langle\beta m \mid q_{\gamma l}\right\rangle=q_{\gamma l}\left\langle\alpha n \mid q_{\gamma l}\right\rangle,
$$

where $q_{\gamma l}$ is the quasienergy eigenvalue and $\left|q_{\gamma l}\right\rangle$ is the corresponding eigenvector, $\left\langle\alpha n \mid q_{\gamma l}\right\rangle=\phi_{\alpha \gamma}^{[n-l]}$. Here, $H_{F}$ is the time-independent Floquet Hamiltonian defined by

$$
\left\langle\alpha n\left|H_{F}\right| \beta m\right\rangle=H_{\alpha \beta}^{[n-m]}+n \hbar \omega \delta_{\alpha \beta} \delta_{n m} .
$$

For simplicity, we set $\hbar=1$ hereafter.

For the effective Hamiltonian of the superconducting flux qubit given by Eq. (4), there are only three nonvanishing Fourier components,

$$
\begin{aligned}
& H^{[0]}=-\frac{1}{2}\left(\begin{array}{cc}
\varepsilon_{0} & \Delta \\
\Delta & -\varepsilon_{0}
\end{array}\right), \\
& H^{[+1]}=-\frac{1}{4}\left(\begin{array}{cc}
A & 0 \\
0 & -A
\end{array}\right), \\
& H^{[-1]}=-\frac{1}{4}\left(\begin{array}{cc}
A & 0 \\
0 & -A
\end{array}\right),
\end{aligned}
$$

where $H^{[n-m]}+n \omega \delta_{n m} I(I: 2 \times 2$ identity matrix) forms the $n$th column and the $m$ th row of the Floquet blocks. The Floquet matrix for the qubit represented by bases of $|\alpha n\rangle$ and $|\beta m\rangle$ according to Eq. (12) is given as follows:

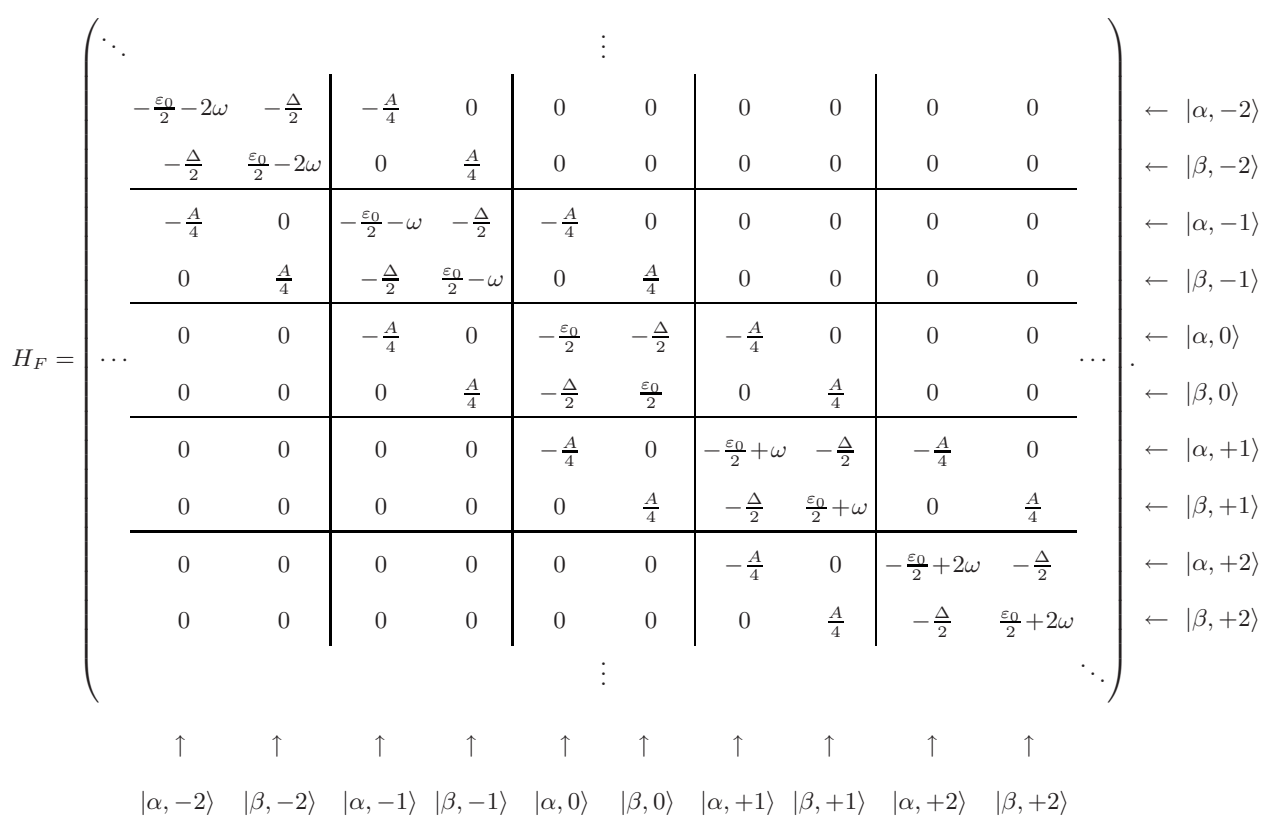

Eigenvalues of this Floquet matrix are numerically solved by truncating the number of the Floquet blocks. For numerical solutions in Sec. IV, 101 Floquet blocks ( $n=-50$ to 50 ) are included to be sufficiently converged for multiphoton processes. It is worthwhile to note that there has been no approximation made in Eq. (14) to solve the time-dependent Hamiltonian of Eq. (4). Therefore, Eq. (14) can be applied for all parameter regimes. 
After solving the eigenvalue problem of the Floquet matrix, the time-averaged transition probability between $|\alpha\rangle$ and $|\beta\rangle$ can be computed by

$$
\bar{P}_{\alpha \rightarrow \beta}=\sum_{n} \sum_{\gamma l}\left|\left\langle\beta n \mid q_{\gamma l}\right\rangle\left\langle q_{\gamma l} \mid \alpha 0\right\rangle\right|^{2},
$$

which is corresponding to the probability of finding the excited state of the qubit in experiment.

\section{ANALYTIC SOLUTION OF THE FLOQUET MATRIX FOR THE DRIVEN QUBIT SYSTEM}

To solve the Floquet matrix of Eq. (14) analytically, we employ the generalized Van Vleck (GVV) nearly degenerate perturbation theory $[16,17]$. By introducing a perturbation parameter, $\lambda=-\Delta / 2$, the Floquet matrix can be divided into unperturbed and perturbed parts,

$$
H_{F}=H_{0}+\lambda V
$$

where $H_{0}$ has the following matrix structure:

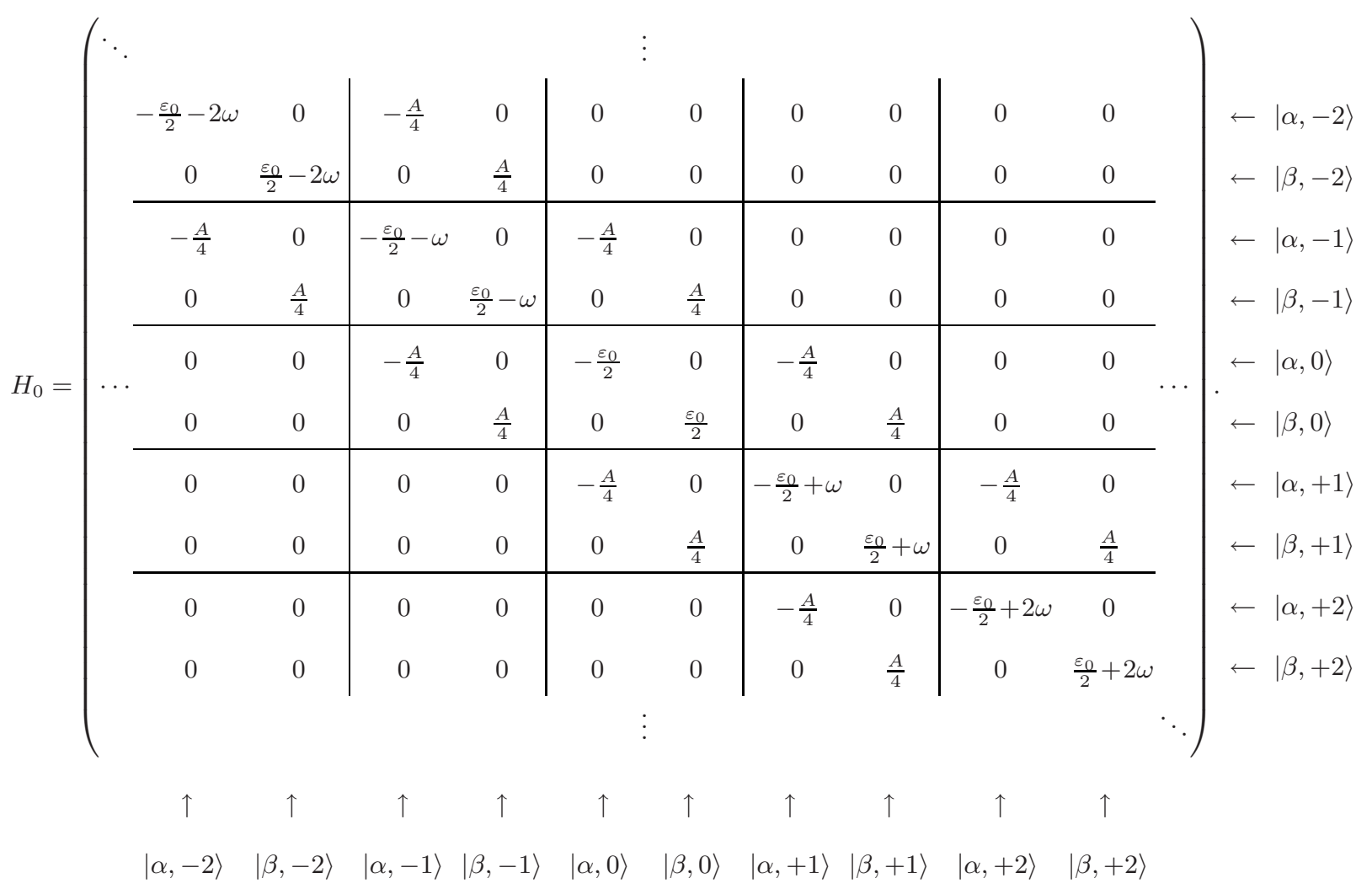

(17)

To obtain the eigenstates of $H_{0}$, Eq. (17) can be separated into two segments because all off-diagonals are zero in each 2 $\times 2$ block, i.e., $|\alpha\rangle$ and $|\beta\rangle$ are decoupled in $H_{0}$,

$$
H_{0}(\alpha \text { or } \beta)=\left(\begin{array}{ccccccc}
\ddots & & & \vdots & & & \\
& b-2 \omega & a & 0 & 0 & 0 & \\
& a & b-\omega & a & 0 & 0 & \\
\cdots & 0 & a & b & a & 0 & \cdots \\
& 0 & 0 & a & b+\omega & a & \\
& 0 & 0 & 0 & a & b+2 \omega & \\
& & & \vdots & & & \ddots
\end{array}\right) \text {, }
$$

where $b=-\varepsilon_{0} / 2$ and $a=-A / 4$ for the state of $|\alpha\rangle$, and $b=+\varepsilon_{0} / 2$ and $a=+A / 4$ for $|\beta\rangle$. Eigenvalues and eigenvectors of Eq. (18) can be analytically solved in terms of Bessel functions $J_{k}(x)$ (see the Appendix A for detailed derivation),

Eigenvalues: $b+n \omega \quad(n$ is an integer $)$, 


$$
\text { Eigenvectors: } \quad \phi_{n}=\sum_{k=-\infty}^{\infty} J_{k-n}\left(-\frac{2 a}{\omega}\right)|k\rangle .
$$

Therefore, the eigenstates of $H_{0}$ in Eq. (17) are represented as follows:

$$
\begin{gathered}
\left|\alpha^{\prime}, n\right\rangle=\sum_{k=-\infty}^{\infty} J_{k-n}\left(\frac{A}{2 \omega}\right)|\alpha k\rangle, \\
\left|\beta^{\prime}, m\right\rangle=\sum_{k=-\infty}^{\infty} J_{k-m}\left(-\frac{A}{2 \omega}\right)|\beta k\rangle .
\end{gathered}
$$

Since the GVV method requires the eigenstates for the unperturbed Hamiltonian, the Floquet matrix $H_{F}$ in Eq. (14) is now rewritten in terms of the bases of $\left|\alpha^{\prime}, n\right\rangle$ and $\left|\beta^{\prime}, m\right\rangle$ (see the Appendix A),

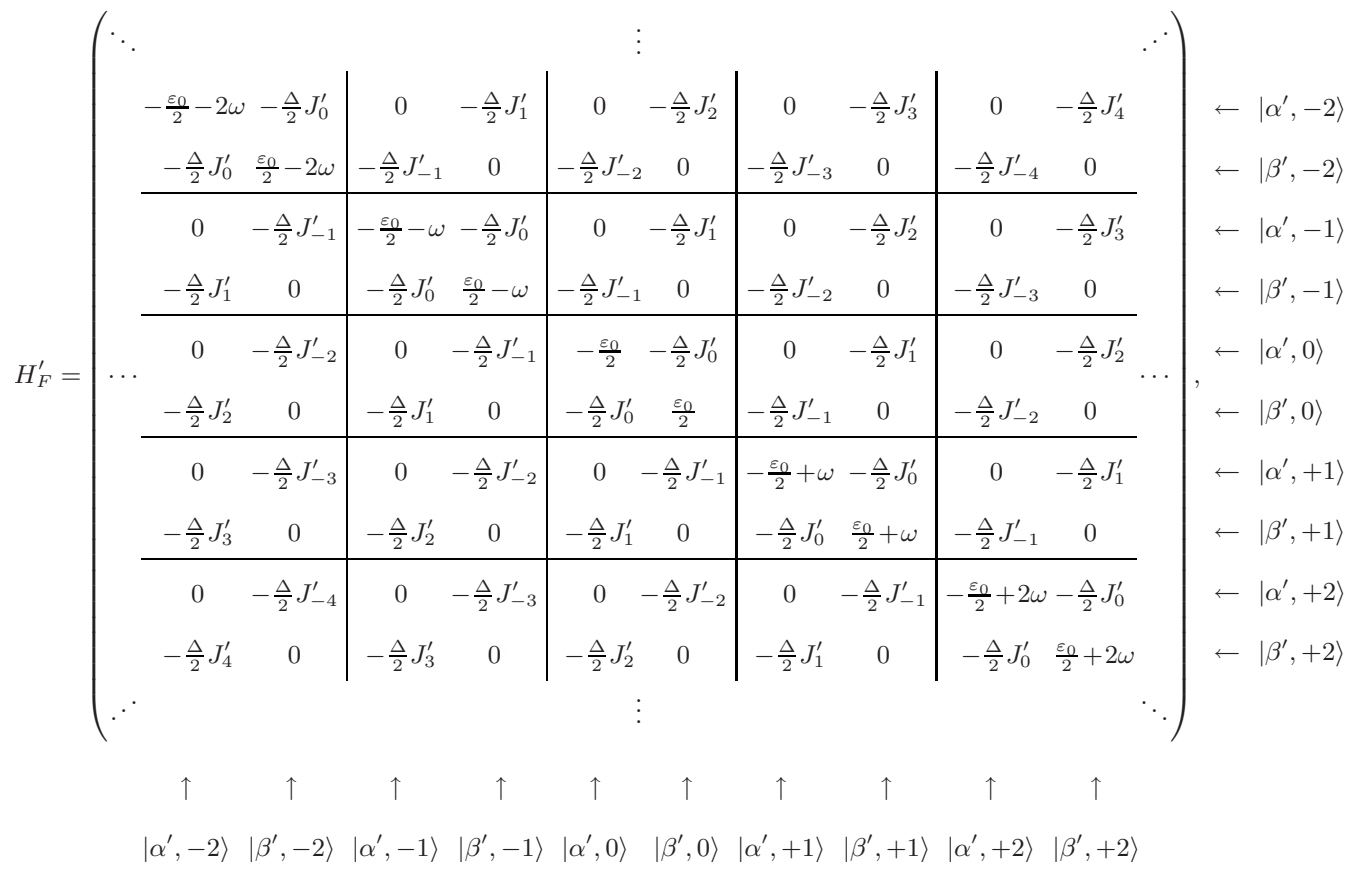

where $J_{k}^{\prime}=J_{k}(A / \omega)$.

From the matrix structure of Eq. (21), one can see that $\left|\alpha^{\prime}, 0\right\rangle$ is coupled to $\left|\beta^{\prime},-n\right\rangle$ throughout an off-diagonal term of $-\frac{\Delta}{2} J_{-n}^{\prime}$. When the Floquet states $\left|\alpha^{\prime}, 0\right\rangle$ and $\left|\beta^{\prime},-n\right\rangle$ are nearly degenerate, namely, $-\varepsilon_{0} / 2 \approx \varepsilon_{0} / 2-n \omega$, Eq. (21) is reduced to a $2 \times 2$ matrix by neglecting all other coupling terms except the one between $\left|\alpha^{\prime}, 0\right\rangle$ and $\left|\beta^{\prime},-n\right\rangle$,

$$
H_{\mathrm{RWA}}=\left(\begin{array}{cc}
-\frac{\varepsilon_{0}}{2} & -\frac{\Delta}{2} J_{-n}^{\prime} \\
-\frac{\Delta}{2} J_{-n}^{\prime} & \frac{\varepsilon_{0}}{2}-n \omega
\end{array}\right) .
$$

This is equivalent to the rotating wave approximation (RWA), whose form is identical to previous works used for the superconducting qubit subject to the longitudinal coupling $[5,19,20]$. Note that this RWA is different from conventional one subject to the transverse coupling, where RWA breaks down in the strong field.

To go beyond RWA, we extend the GVV method allowing the reduction of the infinite-dimensional $H_{F}^{\prime}$ into a $2 \times 2$ ef fective Hamiltonian which includes all $n$-photon coupling channels (see the Appendix B for details),

$$
H_{\mathrm{GVV}}=\left(\begin{array}{cc}
-\frac{\varepsilon_{0}}{2}+\delta & u \\
u & \frac{\varepsilon_{0}}{2}-\delta-n \omega
\end{array}\right),
$$

where $\delta$ is the ac Stark level shift and $u$ is the nonvanishing off-diagonal term which is related to the power broadening of the transition probability peaks,

$$
\begin{aligned}
u= & J_{-n}^{\prime} \lambda-\sum_{\substack{k=-\infty \\
k \neq-n}}^{\infty}\left(\sum_{\substack{l=-\infty \\
l \neq-n}}^{\infty} \frac{J_{k}^{\prime} J_{l}^{\prime} J_{k+l+n}^{\prime}}{\left(\varepsilon_{0}+k \omega\right)\left(\varepsilon_{0}+l \omega\right)}+\frac{J_{k}^{\prime 2} J_{-n}^{\prime}}{\left(\varepsilon_{0}+k \omega\right)^{2}}\right) \lambda^{3} \\
& +O\left(\lambda^{5}\right),
\end{aligned}
$$




$$
\delta=-\sum_{\substack{k=-\infty \\ k \neq-n}}^{\infty} \frac{J_{k}^{\prime 2}}{\varepsilon_{0}+k \omega} \lambda^{2}+O\left(\lambda^{4}\right) .
$$

The leading term appears in the first order of $\lambda(=-\Delta / 2)$ in $u$ and in the second order of $\lambda$ in $\delta$. In other words, most of dominant terms involving all multiphoton resonance processes are taken into account within the second-order perturbation of $\lambda$, in contrast to the conventional transverse coupling case that requires the $(2 n+1)$-st-order perturbation to obtain the nonvanishing terms for $n$-photon process [17]. If one considers the first-order term only, $H_{\mathrm{GVV}}$ is reduced to $H_{\text {RWA }}$. Note that Eqs. (23)-(25) are valid regardless of the strong or weak driving field amplitude $A$.

The effective Hamiltonian of Eq. (23) has the standard form for the two-level system in an oscillating field beyond RWA [10,16-18]. Eigenvalues of Eq. (23) are given by

$$
q_{ \pm}=-\frac{n \omega}{2} \pm p
$$

where

$$
p^{2}=\frac{\left(n \omega-\varepsilon_{0}+2 \delta\right)^{2}}{4}+u^{2}
$$

The $n$-photon time-dependent transition probability from $|\alpha\rangle$ to $|\beta\rangle$ is obtained by

$$
P_{\alpha \rightarrow \beta}^{(n)}(t)=\frac{u^{2}}{p^{2}} \sin ^{2}(p t),
$$

and the $n$-photon time-averaged transition probability is obtained by

$$
\bar{P}_{\alpha \rightarrow \beta}^{(n)}=\lim _{T \rightarrow \infty} \frac{1}{T} \int_{0}^{T} P_{\alpha \rightarrow \beta}^{(n)}(t) d t=\frac{1}{2} \frac{u^{2}}{u^{2}+\left(n \omega-\varepsilon_{0}+2 \delta\right)^{2} / 4} .
$$

With the first-order term only (RWA limit), it yields to

$$
\bar{P}_{\alpha \rightarrow \beta}^{(n)}=\frac{1}{2} \frac{\left(\Delta J_{n}^{\prime}\right)^{2}}{\left(\Delta J_{n}^{\prime}\right)^{2}+\left(n \omega-\varepsilon_{0}\right)^{2}},
$$

since $J_{-n}(x)=(-1)^{n} J_{n}(x)$. This RWA result is identical with previous formulation in Ref. [5]. Note that the first-order expression does not contain the level shift term.

Let us now investigate the behavior of the level shift $\delta$ in the weak and strong driving field regimes (see Appendix $C$ for detailed derivation). The level shift is particularly important in qubit calibration because it determines the accurate positions of resonance peaks. From the second-order GVV in Eq. (25), $\delta$ is proportional to $\Delta^{2}$ and given by the series with the Bessel function involving $A$ as its argument. In the weakfield regime, $\delta$ is approximated as the following:

$$
\begin{aligned}
\delta & \approx-\frac{A^{2} \Delta^{2} \varepsilon_{0}}{8 \omega^{2}\left(\varepsilon_{0}^{2}-\omega^{2}\right)} \quad(n=0), \\
& \approx-\frac{\Delta^{2}}{4 \varepsilon_{0}}+\frac{A^{2} \Delta^{2}\left(\varepsilon_{0}+2 \omega\right)}{16 \omega^{2} \varepsilon_{0}\left(\varepsilon_{0}+\omega\right)} \quad(n=1),
\end{aligned}
$$

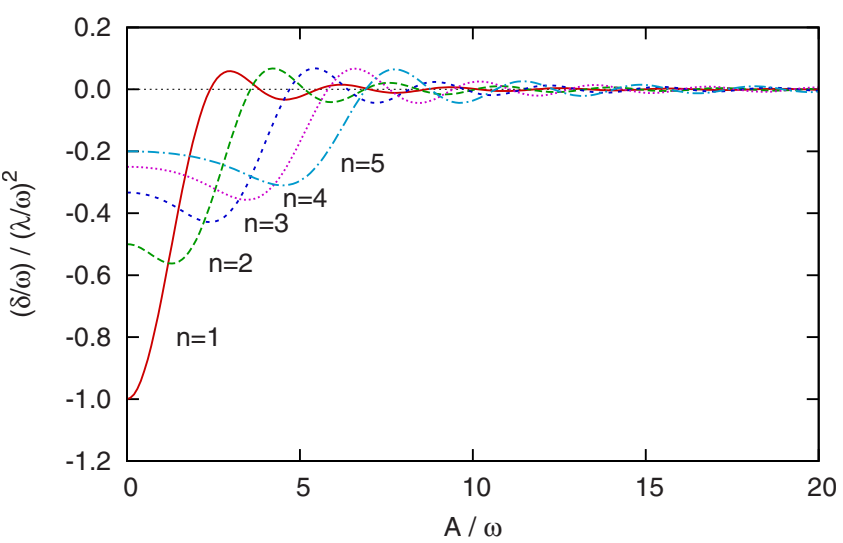

FIG. 3. (Color online) Plots of the level shift $\delta$ as a function of the field amplitude $A$.

$$
\approx-\frac{\Delta^{2}}{4 \varepsilon_{0}}-\frac{\Delta^{2} A^{2}}{8 \varepsilon_{0}\left(\varepsilon_{0}^{2}-\omega^{2}\right)} \quad(n \geqslant 2) .
$$

On the other hand, $\delta$ in the strong-field regime asymptotically becomes zero,

$$
\delta \approx-\frac{\Delta^{2}}{4} \sum_{k=1}^{\infty}\left(\frac{J_{k-n}^{\prime 2}}{k \omega}-\frac{J_{-k-n}^{\prime 2}}{k \omega}\right) \approx 0 .
$$

This is somewhat surprising because the level shift presumably increases as the field amplitude increases like classical examples of the two-level system $[10,11,17,18]$. For this superconducting qubit system, however, longitudinal terms driven by the strong ac field show different behavior of the level shift. The two harmonic series with the Bessel functions in Eq. (32) are diverged separately but canceled by each other because $J_{k-n}^{\prime 2} \approx J_{-k-n}^{\prime 2}$ for all $k$. Therefore, the level shift asymptotically diminishes in the strong-field regime. However, $\delta$ does not monotonously decrease because of the summation with the Bessel functions in Eq. (25). Figure 3 shows the change of $\delta$ [numerically computed from Eq. (25)] as a function of the field amplitude $A$ for a few $n$-photon cases. Due to this level shift $\delta$, the multiphoton resonance condition is not $\varepsilon_{0}=n \omega$ as derived from RWA, but shifted to

$$
\varepsilon_{0}-2 \delta=n \omega \quad(n \geqslant 1) .
$$

To summarize, the level shift $\delta$ appears more vivid in the weak-field regime. Note that the shift $\delta$ is most prominent when the number of photons involved is small $(n \gtrsim 1)$.

\section{RESULTS AND DISCUSSIONS}

Figure 4 shows the quasienergies and corresponding timeaveraged transition probabilities as a function of $\varepsilon_{0}$ with fixed parameters of $\Delta / \omega=0.5$ and $A / \omega=5.0$, computed by solving Eq. (14). The solid red lines indicate lower Floquet states and dashed blue lines indicate upper Floquet states. Due to the periodicity of the quasienergy, the quasienergy plot has repeating structure by $\omega$ with the avoided crossings between the lower and upper Floquet states in the vicinity of $\varepsilon_{0} \approx n \omega$ ( $n$ is a positive integer). At the avoided crossings, 

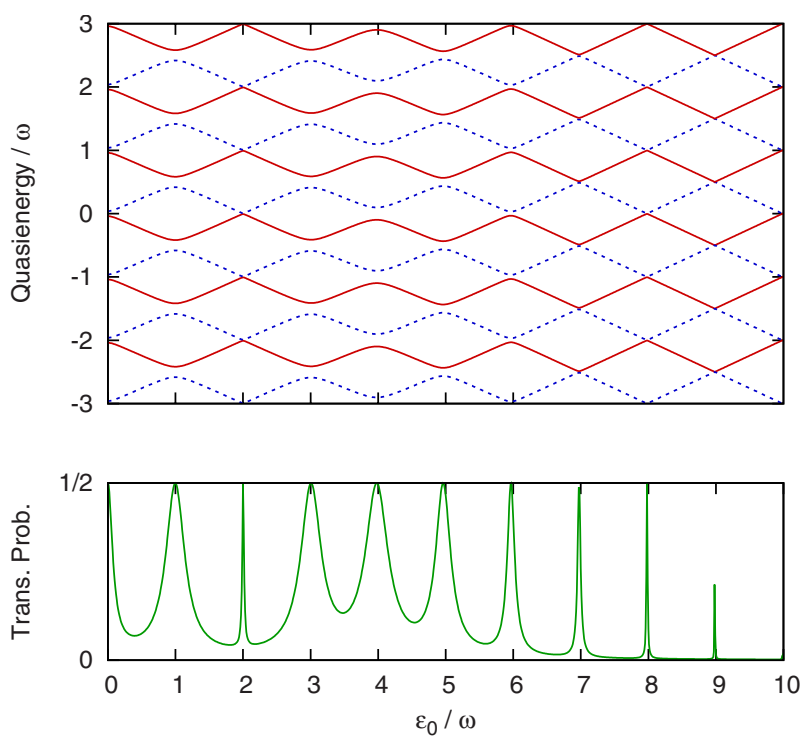

FIG. 4. (Color online) Plots of quasienergies and transition probabilities as a function of $\varepsilon_{0} . \Delta / \omega=0.5$ and $A / \omega=5.0$ are used.

the lower and upper Floquet states are strongly mixed and resonance transitions between $|\alpha\rangle$ and $|\beta\rangle$ occur, as shown in the plot of time-averaged transition probability. Also $\varepsilon_{0}$ $\approx n \omega$ indicates that these transitions are multiphoton resonance processes.

To compare the numerical and analytic results, we present the transition probability plots as a function of $\varepsilon_{0}$ in Fig. 5, computed by a numerical solution of the full matrix of Eq. (14) and analytic solutions of the $2 \times 2$ matrices of Eqs. (22) and (23). Figure 5(a) shows the weak-field case of $A / \omega$ $=0.5$ and Fig. 5(b) the strong-field case of $A / \omega=10.0 . \Delta / \omega$ $=0.5$ is used for both cases. The solid red line indicates the numerical solution by solving a $202 \times 202$ Floquet matrix. The dotted green line is used for the RWA solution, and the dashed blue line for the second-order GVV solution. The third-order GVV result is not shown because it is almost the same as the second-order one in this regime. The analytic GVV expression shows very good agreement with the numerical results near $n$-photon resonance regions, whereas the RWA results show large deviations in the weak-field case [Fig. 5(a)]. The time-averaged transition probability hits the maximum value of $\frac{1}{2}$ near $\varepsilon_{0} \approx n \omega$. Note that in Fig. 5(a) those resonance positions of the numerical and GVV solu-

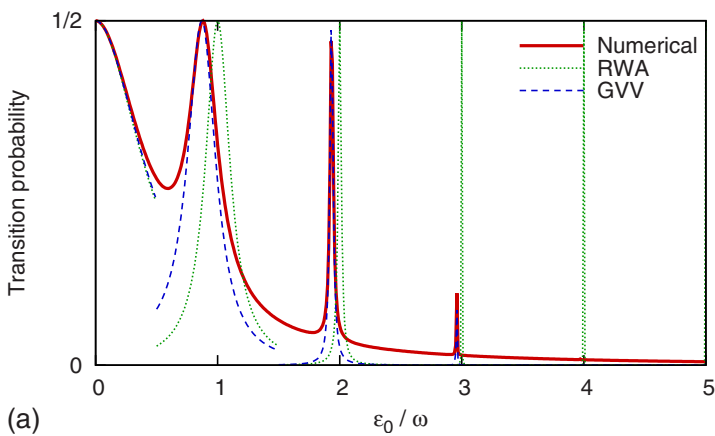

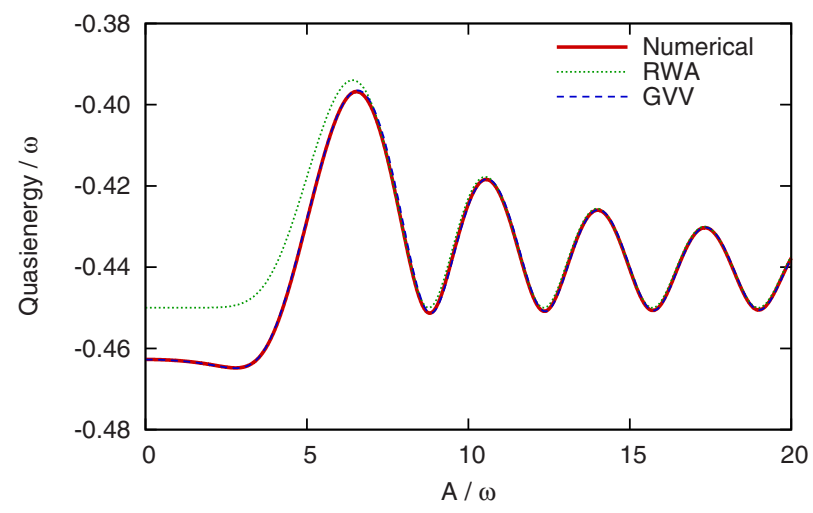

FIG. 6. (Color online) Comparison for numerical results and analytic RWA and GVV results by the quasienergy plots as a function of $A . \Delta / \omega=0.5$ and $\varepsilon_{0} / \omega=4.9$ are used.

tions are ac Stark shifted due to the level shift $\delta$ in the weakfield regime. As $\varepsilon_{0}$ increases, this shift becomes smaller according to the dominant terms in Eq. (31). Since the RWA method does not include the level shift, the dotted green line remains unshifted. On the other hand, in the strong-field case of Fig. 5(b), the level shift diminishes and all lines coincide near the multiphoton resonances.

To reveal the dependence of the ac Stark shift upon the field amplitude, Fig. 6 plots quasienergies as a function of $A$. $\varepsilon_{0} / \omega=4.9$ and $\Delta / \omega=0.5$ are fixed. For clarity, Fig. 6 shows the behavior of the lower quasienergies only. The GVV results (dashed blue line) coincide with the numerical results (solid red line). In contrast, quasienergies computed by the RWA method (dotted green line) deviates from the numerical results in the weak $A$ region and this deviation decreases as $A$ increases. Oscillating patterns as a function of $A$ are mainly due to $|u|$, i.e., the shape of the Bessel function. From Eqs. (26) and (27), $q$ mainly involves $\left|J_{n}(A / \omega)\right|$ when $\varepsilon_{0}-2 \delta$ $\approx n \omega$. For example, in Fig. 6 , the oscillating patterns follow $\left|J_{5}(A / \omega)\right|$ because $\varepsilon_{0} / \omega \approx 5$.

In the transition probability plot of Fig. 4, one can see that the width of the peaks varies nonmonotonically as $\varepsilon_{0}$ (i.e., $n$ ) increases. In fact, these patterns also depend on $A$. These widths are correlated with the difference between the lower and upper states at the avoided crossings in the quasienergy plot. Figures 7(a)-7(c) show a contour map of the transition probability computed by Eq. (15) as a function of $A$ and $\varepsilon_{0}$ with $\Delta / \omega=0.1,0.5$, and 1.0 , respectively. Multiphoton reso-

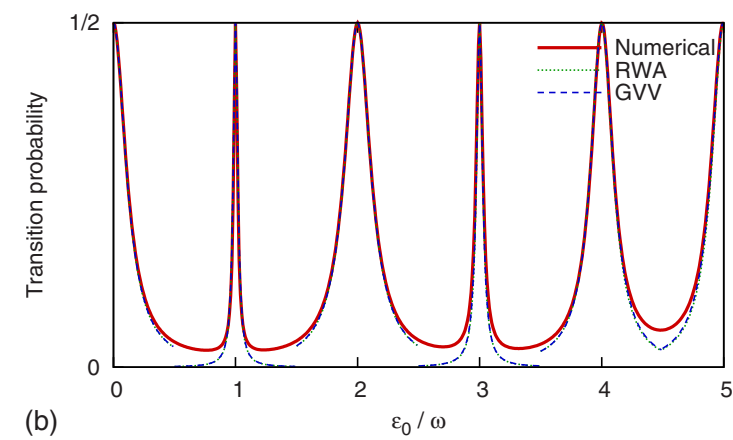

FIG. 5. (Color online) Comparison for numerical results and analytic RWA and GVV results by the transition probability plots as a function of $\varepsilon_{0} . \Delta / \omega=0.5$ is used. (a) Weak field: $A / \omega=0.5$. (b) Strong field: $A / \omega=10.0$. 

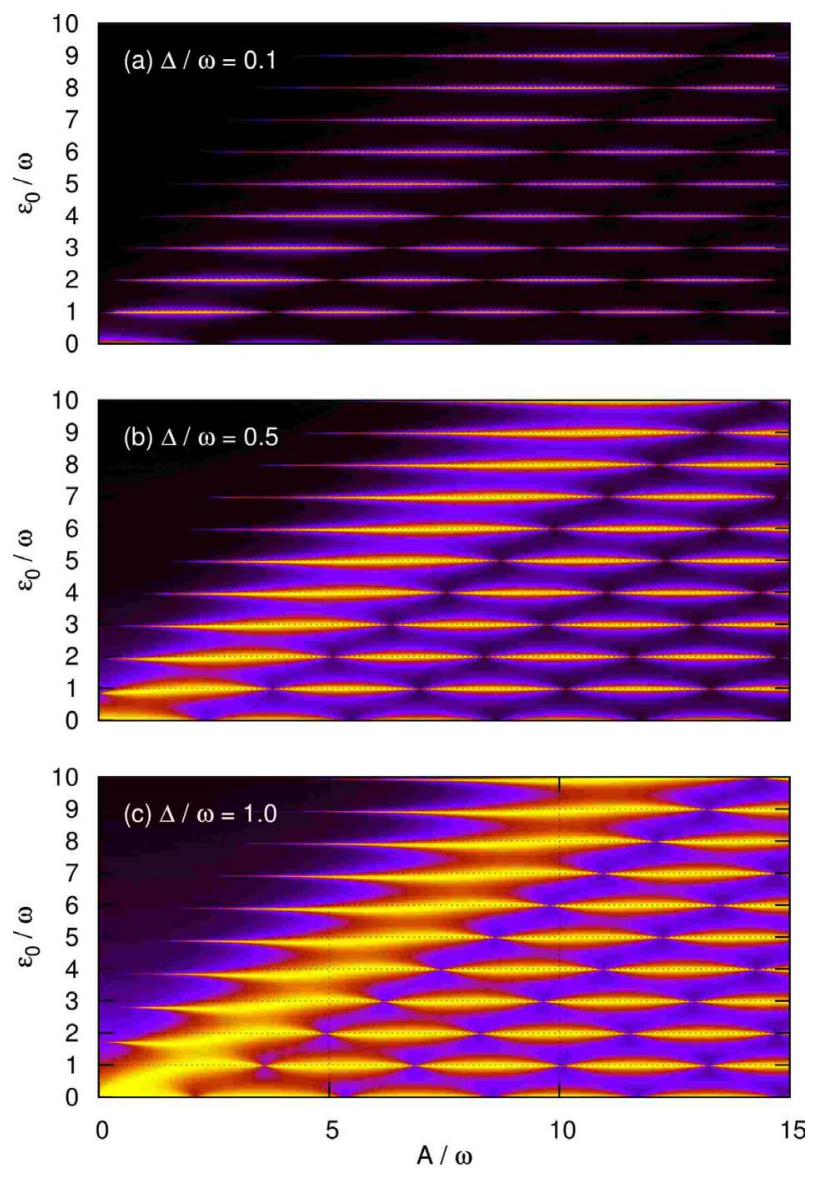

Trans. Prob.

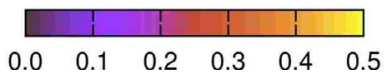

FIG. 7. (Color online) A contour map of the transition probabilities as a function of $A$ and $\varepsilon_{0}$ with different values of $\Delta$. (a) $\Delta / \omega$ $=0.1$, (b) $\Delta / \omega=0.5$, and (c) $\Delta / \omega=1.0$.

nance processes occur near integer numbers closest to $\varepsilon_{0} / \omega$ indicated as bright yellow fringes in the plot. Figure 7(a) with relatively small $\Delta$ agrees well with previous experimental and theoretical results for the superconducting flux qubit [5]. In Figs. 7(b) and 7(c) with large $\Delta$, however, it is clearly shown that the level shift alters multiphoton resonance positions especially in the regime of small $\varepsilon_{0}$ and weak $A$. Also for all figures resonance transitions are suppressed at certain positions of $A$, which is a phenomenon known as the coherent destruction of tunneling [26]. These positions can be determined by the roots of the Bessel function of order of $n$ that is the nearest integer to $\varepsilon_{0} / \omega[5,20]$. From the analytic expression of the time-averaged transition probability in Eq. (29), we can easily confirm that $\bar{P}_{\alpha \rightarrow \beta}^{(n)} \rightarrow 0$ as $u \rightarrow 0$. When $J_{-n}(A / \omega)=0$, the first order of $\Delta$ in $u$ in Eq. (24) disappears and $u$ is proportional to $\Delta^{3}$. Thus, the width of the timeaveraged transition probability becomes very narrow near the roots of the Bessel function, $J_{-n}(A / \omega)$, which depends on the number of photons $n\left(\approx \varepsilon_{0} / \omega\right)$ and the amplitude $A$.

\section{CONCLUSION}

In this paper, we extend the Floquet theory to investigate the superconducting flux qubit driven by a strong ac field.
The numerical and analytic solutions of the generalized Floquet formalism are applied to explain multiphoton resonance processes between the qubit and the driving ac field. We compare two analytic solutions: the rotating wave approximation (RWA) and the generalized Van Vleck (GVV) nearly degenerate perturbation theory. It is shown that the GVV approach accurately includes the ac Stark level shift which is completely absent within RWA. The level shifts in resonance positions are significant when the tunnel splitting is large, the field is weak, and a small number of photons are involved. The quasienergy and the time-averaged transition probability from Floquet calculations are plotted showing multiphoton resonance transitions at the avoided crossings. From the transition probability plot as a function of the field amplitude and the detuning energy, we observe the Bessel functionlike fringe patterns including the level shift. The general method described here provides a unified theoretical treatment covering a wide range of parameter space. Applications of the Floquet theory to various superconducting qubits lead us to a better understanding of the results of spectroscopy measurement and the dynamics of ac driven qubits which are important for more accurate characterization of and performance improvement of the qubits.

\section{ACKNOWLEDGMENTS}

This work was supported in part by the NSF (Contract No. DMR-0325551). S.I.C. was partially supported by the NSF (Contract No. PHY-0757194), and by the Chemical Sciences, Geosciences and Biosciences Division of the Office of Basic Energy Sciences, Office of Sciences, U.S. Department of Energy.

\section{APPENDIX A: FLOQUET MATRIX REPRESENTATION}

We start from the eigenvalue problem of the infinitedimensional tridiagonal matrix in Eq. (18). It can be considered as a Floquet matrix transformed from a function $h(t)$ $=b+2 a \cos \omega t$ with Fourier bases of $|k\rangle$ ( $k$ is an integer), where $a$ and $b$ are given numbers. After an inverse transformation, the original equation to be solved is the following eigenvalue problem:

$$
\left(h(t)-i \frac{\partial}{\partial t}\right) \phi(t)=\lambda \phi(t) .
$$

A trivial solution is $\lambda=b$. Its eigenfunction is given by a Fourier expansion with Bessel functions [27],

$$
\phi(t)=e^{-i(2 a / \omega) \sin \omega t}=\sum_{k=-\infty}^{\infty} J_{k}\left(-\frac{2 a}{\omega}\right) e^{i k \omega t} .
$$

For eigenvalues of $\lambda_{n}=b+n \omega$ ( $n$ is an integer), $\phi_{n}(t)$ is solved as

$$
\begin{aligned}
\phi_{n}(t) & =e^{i n \omega t} e^{-i(2 a / \omega) \sin \omega t}=\sum_{k=-\infty}^{\infty} J_{k}\left(-\frac{2 a}{\omega}\right) e^{i(n+k) \omega t} \\
& =\sum_{k=-\infty}^{\infty} J_{k-n}\left(-\frac{2 a}{\omega}\right) e^{i k \omega t} .
\end{aligned}
$$

It proves the eigensolutions of Eq. (19). 
Next, let us construct the Floquet matrix of Eq. (21) with bases of $\left|\alpha^{\prime}, n\right\rangle$ and $\left|\beta^{\prime}, m\right\rangle$ in Eq. (20). Let $z=A / 2 \omega$. From $\left\langle\alpha k\left|H_{F}\right| \beta l\right\rangle=-\frac{\Delta}{2} \delta_{k l}$ in Eq. (14) and the addition theorem for Bessel function $J_{n}(y+z)=\Sigma_{m} J_{m}(y) J_{n-m}(z)$ for any integer $n$ [27], one can obtain

$$
\begin{aligned}
\left\langle\alpha^{\prime}, n\left|H_{F}\right| \beta^{\prime}, m\right\rangle & =\sum_{k=-\infty}^{\infty} \sum_{l=-\infty}^{\infty} J_{k-n}(z) J_{l-m}(-z)\left\langle\alpha k\left|H_{F}\right| \beta l\right\rangle \\
& =-\frac{\Delta}{2} \sum_{k=-\infty}^{\infty} J_{k-n}(z) J_{-k+m}(z)=-\frac{\Delta}{2} J_{m-n}(2 z),
\end{aligned}
$$

$$
\begin{aligned}
\left\langle\beta^{\prime}, n\left|H_{F}\right| \alpha^{\prime}, m\right\rangle & =\sum_{k=-\infty}^{\infty} \sum_{l=-\infty}^{\infty} J_{k-n}(-z) J_{l-m}(z)\left\langle\beta k\left|H_{F}\right| \alpha l\right\rangle \\
& =-\frac{\Delta}{2} \sum_{k=-\infty}^{\infty} J_{-k+n}(z) J_{k-m}(z)=-\frac{\Delta}{2} J_{n-m}(2 z) .
\end{aligned}
$$

From $\left\langle\alpha k\left|H_{F}\right| \alpha l\right\rangle=\left(-\frac{\varepsilon_{0}}{2}+k \omega\right) \delta_{k l}-\frac{A}{4}\left(\delta_{k, l+1}+\delta_{k, l-1}\right)$ in Eq. (14), a recursive relation for Bessel function $J_{n-1}(z)+J_{n+1}(z)$ $=\frac{2 n}{z} J_{n}(z) \quad[27], \quad$ and summation of $\sum_{m} J_{m}(z) J_{m-n}(z)$ $=\Sigma_{m} J_{m}(z) J_{-m+n}(-z)=J_{n}(0)=\delta_{n 0}$ for any integer $n$, one can obtain

$$
\begin{aligned}
\left\langle\alpha^{\prime}, n\left|H_{F}\right| \alpha^{\prime}, m\right\rangle= & \sum_{k=-\infty}^{\infty} \sum_{l=-\infty}^{\infty} J_{k-n}(z) J_{l-m}(z)\left\langle\alpha k\left|H_{F}\right| \alpha l\right\rangle \\
= & \sum_{k=-\infty}^{\infty} J_{k-n}(z)\left[\left(-\frac{\varepsilon_{0}}{2}+k \omega\right) J_{k-m}(z)\right. \\
& \left.-\frac{A}{4}\left\{J_{k-m-1}(z)+J_{k-m+1}(z)\right\}\right] \\
= & \sum_{k=-\infty}^{\infty} J_{k-n}(z)\left(-\frac{\varepsilon_{0}}{2}+k \omega-(k-m) \omega\right) J_{k-m}(z) \\
= & \left(-\frac{\varepsilon_{0}}{2}+m \omega\right) \sum_{k=-\infty}^{\infty} J_{k-n}(z) J_{k-m}(z) \\
= & \left(-\frac{\varepsilon_{0}}{2}+n \omega\right) \delta_{n m} .
\end{aligned}
$$

Likewise, from $\left\langle\beta k\left|H_{F}\right| \beta l\right\rangle=\left(\frac{\varepsilon_{0}}{2}+k \omega\right) \delta_{k l}+\frac{A}{4}\left(\delta_{k, l+1}+\delta_{k, l-1}\right)$,

$$
\left\langle\beta^{\prime}, n\left|H_{F}\right| \beta^{\prime}, m\right\rangle=\left(\frac{\varepsilon_{0}}{2}+n \omega\right) \delta_{n m} .
$$

Equations from (A4) to (A7) constitute all Floquet matrix elements of Eq. (21).

APPENDIX B: THE $2 \times 2$ EFFECTIVE FLOQUET MATRIX DETERMINED BY THE GVV METHOD

With the perturbation parameter $\lambda=-\Delta / 2$, the Floquet matrix of Eq. (21) is divided into

$$
H_{F}^{\prime}=H_{0}^{\prime}+\lambda V^{\prime} \text {, }
$$

where

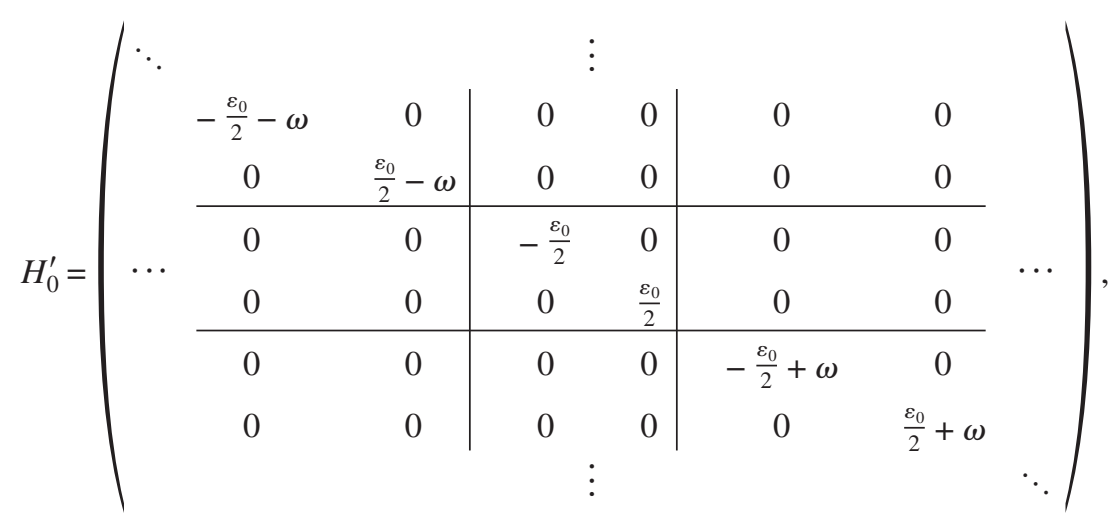

and 


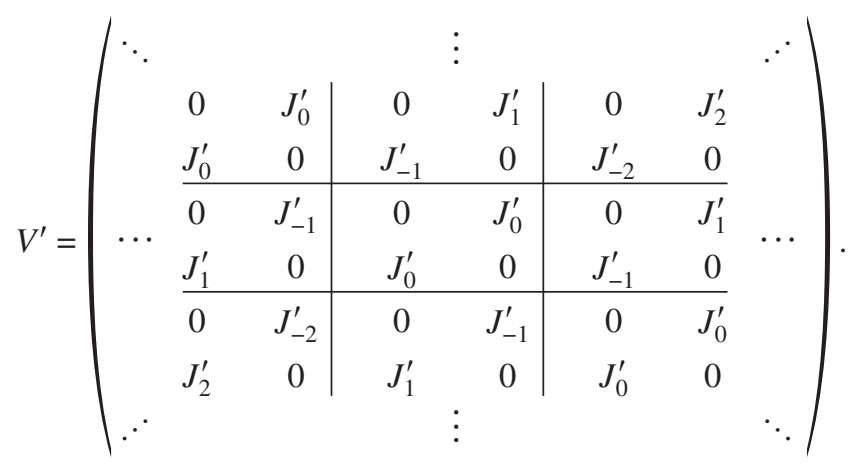

The aim is to reduce the infinite-dimensional Floquet matrix of Eq. (21) into the $2 \times 2$ matrix by the use of the nearly degenerate perturbation formalism in the GVV method $[16,17]$. According to the perturbation theory, the $2 \times 2$ matrix $\mathbf{h}$ and its eigenstate solution $\boldsymbol{\phi}$ can be expanded in powers of $\lambda$,

$$
\begin{aligned}
& \mathbf{h}=\sum_{m=0}^{\infty} \lambda^{m} \mathbf{h}^{(m)}, \\
& \boldsymbol{\phi}=\sum_{m=0}^{\infty} \lambda^{m} \boldsymbol{\phi}^{(m)} .
\end{aligned}
$$

For $n$-photon resonance, the Floquet states $\left|\alpha^{\prime}, 0\right\rangle$ and $\mid \beta^{\prime}$, $-n\rangle$ are nearly degenerate. Thus, the zeroth-order $\boldsymbol{\phi}^{(0)}$ $=\left(\phi_{-}^{(0)} \phi_{+}^{(0)}\right)^{T}$ is given by

$$
\phi_{-}^{(0)}=\left|\alpha^{\prime}, 0\right\rangle \quad \text { and } \quad \phi_{+}^{(0)}=\left|\beta^{\prime},-n\right\rangle .
$$

The zeroth-order $\mathbf{h}^{(0)}$ represented by $\phi_{-}^{(0)}$ and $\phi_{+}^{(0)}$ is given by

$$
\mathbf{h}^{(0)}=\left(\begin{array}{cc}
-\frac{\varepsilon_{0}}{2} & 0 \\
0 & \frac{\varepsilon_{0}}{2}-n \omega
\end{array}\right),
$$

where two eigenstates are nearly degenerate, i.e., $\varepsilon_{0} \approx n \omega$. Following the GVV method, a few high-order terms can be computed as follows:

$$
\begin{gathered}
\phi_{-}^{(1)}=\sum_{\substack{k=-\infty \\
k \neq-n}}^{\infty} \frac{-J_{k}^{\prime}}{\varepsilon_{0}+k \omega}\left|\beta^{\prime}, k\right\rangle, \\
\phi_{+}^{(1)}=\sum_{\substack{k=-\infty \\
k \neq-n}}^{\infty} \frac{J_{k}^{\prime}}{\varepsilon_{0}+k \omega}\left|\alpha^{\prime},-n-k\right\rangle, \\
\mathbf{h}^{(1)}=\left\langle\boldsymbol{\phi}^{(0)}\left|V^{\prime}\right| \boldsymbol{\phi}^{(0)}\right\rangle=J_{-n}^{\prime}\left(\begin{array}{ll}
0 & 1 \\
1 & 0
\end{array}\right),
\end{gathered}
$$

$$
\begin{aligned}
\mathbf{h}^{(2)} & =\left\langle\boldsymbol{\phi}^{(0)}\left|V^{\prime}\right| \boldsymbol{\phi}^{(1)}\right\rangle-\mathbf{h}^{(1)}\left\langle\boldsymbol{\phi}^{(0)} \mid \boldsymbol{\phi}^{(1)}\right\rangle \\
& =\sum_{\substack{k=-\infty \\
k \neq-n}}^{\infty} \frac{J_{k}^{\prime 2}}{\varepsilon_{0}+k \omega}\left(\begin{array}{cc}
-1 & 0 \\
0 & 1
\end{array}\right),
\end{aligned}
$$

$$
\begin{aligned}
\mathbf{h}^{(3)} & =\left\langle\boldsymbol{\phi}^{(1)}\left|V^{\prime}\right| \boldsymbol{\phi}^{(1)}\right\rangle-\left\langle\boldsymbol{\phi}^{(1)} \mid \boldsymbol{\phi}^{(1)}\right\rangle \mathbf{h}^{(1)} \\
& =-\sum_{\substack{k=-\infty \\
k \neq-n}}^{\infty}\left(\sum_{\substack{l=-\infty \\
l \neq-n}}^{\infty} \frac{J_{k}^{\prime} J_{l}^{\prime} J_{k+l+n}^{\prime}}{\left(\varepsilon_{0}+k \omega\right)\left(\varepsilon_{0}+l \omega\right)}\right. \\
& \left.+\frac{J_{k}^{\prime 2} J_{-n}^{\prime}}{\left(\varepsilon_{0}+k \omega\right)^{2}}\right)\left(\begin{array}{ll}
0 & 1 \\
1 & 0
\end{array}\right) .
\end{aligned}
$$

Equations (B9) and (B11) form the first- and third-order terms of $u$ in Eq. (24), respectively, and Eq. (B10) forms the second-order term of $\delta$ in Eq. (25).

\section{APPENDIX C: ASYMPTOTIC EXPRESSIONS OF THE LEVEL SHIFT}

For small arguments $(x \ll 1)$, the Bessel function has the following asymptotic form:

$$
J_{k}(x) \approx \frac{1}{k !}\left(\frac{x}{2}\right)^{k} \quad(k \geqslant 0),
$$

where $x=A / \omega$. Thus, in the weak-field regime, $J_{0}^{\prime}$ is dominant over $J_{k}^{\prime}(k \neq 0)$. When only $J_{0}^{\prime}$ is included in the sum of Eq. (25), $\delta$ is approximated as

$$
\delta=-\frac{\Delta^{2}}{4 \varepsilon_{0}}+O\left(A^{2}, \Delta^{4}\right) \quad(n \geqslant 1) .
$$

For nonphoton-assisted process $(n=0)$, there is no $\Delta^{2}$ term in $\delta$, i.e., $\delta \approx 0$ because $J_{0}^{\prime}$ is excluded in the sum of Eq. (25). If we approximate $\delta$ up to $O\left(A^{4}\right), \quad J_{0}^{\prime}\left(\approx 1-A^{2} / 4 \omega^{2}\right)$ and $J_{ \pm 1}^{\prime}(\approx \pm A / 2 \omega)$ can be included in the sum of Eq. (25), which yields to 


$$
\begin{aligned}
\delta & =-\frac{A^{2} \Delta^{2} \varepsilon_{0}}{8 \omega^{2}\left(\varepsilon_{0}^{2}-\omega^{2}\right)}+O\left(A^{4}, \Delta^{4}\right) \quad(n=0) \quad(\mathrm{C} 3 \mathrm{a}) \\
& =-\frac{\Delta^{2}}{4 \varepsilon_{0}}+\frac{A^{2} \Delta^{2}\left(\varepsilon_{0}+2 \omega\right)}{16 \omega^{2} \varepsilon_{0}\left(\varepsilon_{0}+\omega\right)}+O\left(A^{4}, \Delta^{4}\right) \quad(n=1) \\
& =-\frac{\Delta^{2}}{4 \varepsilon_{0}}-\frac{\Delta^{2} A^{2}}{8 \varepsilon_{0}\left(\varepsilon_{0}^{2}-\omega^{2}\right)}+O\left(A^{4}, \Delta^{4}\right) \quad(n \geqslant 2) .
\end{aligned}
$$

(C3c)

On the other hand, for large arguments $(x \rightarrow \infty)$, the Bessel function asymptotically becomes

$$
J_{k}(x) \approx \sqrt{\frac{2}{\pi x}} \cos \left(x-\frac{k \pi}{2}-\frac{\pi}{4}\right),
$$

and then $J_{k}^{\prime 2}$ is approximated as

$$
\begin{aligned}
J_{k}^{\prime 2} & \approx \frac{2 \omega}{\pi A} \cos ^{2}\left(\frac{A}{\omega}-\frac{k \pi}{2}-\frac{\pi}{4}\right) \\
& =\frac{\omega}{\pi A}\left[1+\cos \left(\frac{2 A}{\omega}-k \pi-\frac{\pi}{2}\right)\right] \\
& =\frac{\omega}{\pi A}\left[1+(-1)^{k} \sin \left(\frac{2 A}{\omega}\right)\right] .
\end{aligned}
$$

Thus it yields to $J_{k}^{\prime 2} \approx J_{-k}^{\prime 2}$. Using this asymptotic expression as well as $\varepsilon_{0} \approx n \omega, \delta$ in the strong-field regime becomes zero as shown below,

$$
\begin{aligned}
\delta & \approx-\frac{\Delta^{2}}{4} \sum_{\substack{k=-\infty \\
k \neq-n}}^{\infty} \frac{J_{k}^{\prime 2}}{(n+k) \omega}=-\frac{\Delta^{2}}{4} \sum_{\substack{k=-\infty \\
k \neq 0}}^{\infty} \frac{J_{k-n}^{\prime 2}}{k \omega} \\
& =-\frac{\Delta^{2}}{4} \sum_{k=1}^{\infty}\left(\frac{J_{k-n}^{\prime 2}}{k \omega}-\frac{J_{-k-n}^{\prime 2}}{k \omega}\right) \approx 0 .
\end{aligned}
$$

[1] J. R. Friedman, V. Patel, W. Chen, S. K. Tolpygo, and J. E. Lukens, Nature (London) 406, 43 (2000).

[2] M. A. Nielsen and I. L. Chuang, Quantum Computation and Quantum Information, 1st ed. (Cambridge University Press, Cambridge, 2000).

[3] D. Vion, A. Aassime, A. Cottet, P. Joyez, H. Pothier, C. Urbina, D. Esteve, and M. H. Devoret, Science 296, 886 (2002);Y. Yu, S. Han, X. Chu, S. I. Chu, and Z. Wang, ibid. 296, 889 (2002).

[4] I. Chiorescu, Y. Nakamura, C. J. P. M. Harmans, and J. E. Mooij, Science 299, 1869 (2003).

[5] W. D. Oliver, Y. Yu, J. C. Lee, K. K. Berggren, L. S. Levitov, and T. P. Orlando, Science 310, 1653 (2005).

[6] D. M. Berns, W. D. Oliver, S. O. Valenzuela, A. V. Shytov, K. K. Berggren, L. S. Levitov, and T. P. Orlando, Phys. Rev. Lett. 97, 150502 (2006).

[7] J. M. Martinis et al., Phys. Rev. Lett. 95, 210503 (2005).

[8] J. M. Martinis, S. Nam, J. Aumentado, and C. Urbina, Phys. Rev. Lett. 89, 117901 (2002).

[9] F. Yoshihara, K. Harrabi, A. O. Niskanen, Y. Nakamura, and J. S. Tsai, Phys. Rev. Lett. 97, 167001 (2006).

[10] S. I. Chu and D. A. Telnov, Phys. Rep. 390, 1 (2004).

[11] S. I. Chu and W. P. Reinhardt, Phys. Rev. Lett. 39, 1195 (1977).

[12] X. Chu and S. I. Chu, Phys. Rev. A 63, 013414 (2000).
[13] D. A. Telnov and S. I. Chu, Phys. Rev. A 71, 013408 (2005).

[14] T. S. Ho, S. I. Chu, and J. V. Tietz, Chem. Phys. Lett. 96, 464 (1983).

[15] S.-K. Son and S. I. Chu, Phys. Rev. A 77, 063406 (2008).

[16] T. S. Ho and S. I. Chu, Phys. Rev. A 32, 377 (1985).

[17] P. K. Aravind and J. O. Hirschfelder, J. Chem. Phys. 88, 4788 (1984).

[18] J. H. Shirley, Phys. Rev. 138, B979 (1965).

[19] Y. Nakamura, Y. A. Pashkin, and J. S. Tsai, Phys. Rev. Lett. 87, 246601 (2001).

[20] S. Ashhab, J. R. Johansson, A. M. Zagoskin, and F. Nori, Phys. Rev. A 75, 063414 (2007).

[21] Y. S. Greenberg, Phys. Rev. B 76, 104520 (2007).

[22] C. M. Wilson, T. Duty, F. Persson, M. Sandberg, G. Johansson, and P. Delsing, Phys. Rev. Lett. 98, 257003 (2007).

[23] S. Han, J. Lapointe, and J. E. Lukens, Phys. Rev. Lett. 63, 1712 (1989).

[24] A. J. Leggett, S. Chakravarty, A. T. Dorsey, M. P. A. Fisher, A. Garg, and W. Zwerger, Rev. Mod. Phys. 59, 1 (1987).

[25] G. Floquet, Ann. Sci. Ec. Normale Super. 12, 47 (1883).

[26] F. Grossmann, T. Dittrich, P. Jung, and P. Hänggi, Phys. Rev. Lett. 67, 516 (1991).

[27] Handbook of Mathematical Functions, 10th ed., edited by M. Abramowitz and I. A. Stegun (Dover, New York, 1972). 